\title{
EFEITOS DE AGENTES OXIDANTES E OXIGÊNIO DISSOLVIDO NA DESCOLORAÇÃO DO AZO CORANTE ACID ORANGE 7 POR FOTÓLISE E FOTOCATÁLISE
}

\author{
Adriana Campano Lucilha e Keiko Takashima* \\ Departamento de Química, Centro de Ciências Exatas, Universidade Estadual de Londrina, CP 6001, 86051-990 Londrina - PR, \\ Brasil
}

Recebido em 5/5/08; aceito em 23/1/09; publicado na web em 28/7/09

\begin{abstract}
EFFECTS OF OXIDANT AGENTS AND DISSOLVED OXYGEN ON THE DECOLORIZATION OF ACID ORANGE 7 AZO DYE BY PHOTOLYSIS AND PHOTOCATALYSIS. The decolorization of acid orange 7 azo dye by photolysis and photocatalysis by $\mathrm{ZnO}$ was investigated in the presence of oxidants such as $\mathrm{NaClO}_{3}, \mathrm{NaBrO}_{3}, \mathrm{NaIO}_{4}$, and $\mathrm{K}_{2} \mathrm{~S}_{2} \mathrm{O}_{8}$ in an open reactor at $30{ }^{\circ} \mathrm{C}$. The decolorization was relatively fast at lower oxidants concentrations and slow rate at larger concentrations, except for persulfate in the photocatalysis. Concerning photolysis the rate constant enhanced gradually, except for chlorate, outreaching the obtained values by photocatalysis, at higher concentrations. The air saturation decreased the rate constant in both processes and indicated that the azo dye can be decolorized without dissolved oxygen in persulfate medium.
\end{abstract}

Keywords: acid orange 7; photolysis; photocatalysis.

\section{INTRODUÇÃO}

As indústrias de cosméticos, de couro, de papel, têxteis, entre outras, utilizam grandes quantidades de corantes e consomem água limpa nos processos de produção, retornando ao ambiente como efluente. Dentro deste contexto, o setor têxtil gera grandes volumes de efluentes com elevado teor de matéria orgânica e de aditivos usados no processamento. Estes efluentes apresentam coloração intensa, porque aproximadamente $15 \%$ da produção são perdidos, para o meio ambiente, durante a etapa de tingimento das fibras, além dos produtos de degradação altamente carcinogênicos provenientes dos azocorantes, como as aminas aromáticas. ${ }^{1-3}$ Assim, o descarte dos efluentes coloridos no ecossistema é uma fonte dramática de poluição estética, de eutrofização e de perturbações na vida aquática. A necessidade por tratamentos efetivos dos efluentes vem-se tornando uma preocupação crescente para minimizar os problemas ambientais., ${ }^{4,5}$

Os desenvolvimentos de tratamentos químicos têm mostrado que os processos de oxidação avançados (POAs) representam alternativas para redução de subprodutos e cor desses efluentes. Estes processos são baseados na geração do radical hidroxila $\mathrm{HO}^{*}$ altamente reativo com potencial de oxidação de $2,80 \mathrm{~V},{ }^{6}$ capazes de degradar substâncias orgânicas presentes em efluentes industriais. ${ }^{7-9}$ Dentre os POAs, os semicondutores do tipo-n catalisam sob condições normais a degradação e a mineralização dos poluentes orgânicos na presença de radiação UV com energia igual ou maior que a energia de band-gap. ${ }^{6}$ No decorrer das duas últimas décadas, uma grande quantidade de pesquisas tem sido realizada com a finalidade de encontrar meios para purificação de águas e remediação de ambientes aquáticos contaminados com herbicidas, corantes, surfactantes etc. ${ }^{10-12}$ Neste aspecto, o dióxido de titânio e o óxido de zinco têm sido utilizados recentemente como fotocatalisadores efetivos, de baixo custo e atóxicos para a degradação de inúmeros corantes sintéticos. ${ }^{13-18}$

Em nossos trabalhos recentes, a degradação fotocatalítica de herbicidas imidazolinonas foi investigada usando o dióxido de titânio

*e-mail: keiko@uel.br em suspensão aquosa na presença de oxidantes inorgânicos como peróxido de hidrogênio, ${ }^{19-21}$ persulfato de potássio ${ }^{21,22}$ e periodato de sódio. ${ }^{21}$ Com base nestes estudos, constatou-se que o persulfato e o periodato apresentaram maior eficiência na degradação desses herbicidas, quando comparada ao peróxido de hidrogênio. Neste trabalho investigamos a descoloração de acid orange 7 (AO7), um mono azocorante (Figura 1), sob condições nas quais oxidantes inorgânicos, como clorato de sódio, bromato de sódio, periodato de sódio e persulfato de potássio, foram adicionados respectivamente em solução e em suspensão aquosa de óxido de zinco em reator aberto à atmosfera em pH natural sob radiação UV a $30^{\circ} \mathrm{C}$. Além disso, o efeito do oxigênio dissolvido na remoção de cor de acid orange 7 foi investigado, comparando-se as constantes de velocidade de descoloração de $\mathrm{AO} 7$ na presença de persulfato, sob fotocatálise e fotólise, saturando-se ou não com ar, com $\mathrm{O}_{2} \mathrm{e} \mathrm{N}_{2}$, respectivamente, em reator fechado e aberto à atmosfera.

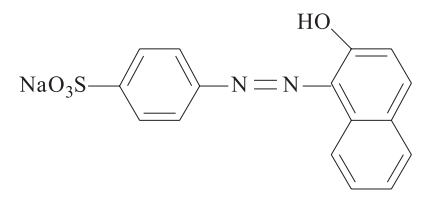

Figura 1. Estrutura química do azo corante acid orange 7 (AO7)

\section{PARTE EXPERIMENTAL}

\section{Materiais}

O azo corante acid orange 7, $\mathrm{AO} 7\left(\mathrm{C}_{16} \mathrm{H}_{11} \mathrm{~N}_{2} \mathrm{SO}_{4} \mathrm{Na}\right)$ - C.I. 15510 , foi gentilmente cedido pela empresa Chimical S.A. O óxido de zinco, $\mathrm{ZnO}$ (Carlo Erba, PA) foi usado como fotocatalisador. Ambos os reagentes foram utilizados sem prévia purificação. Todos os outros reagentes como clorato de sódio $\left(\mathrm{NaClO}_{3}\right.$, Vetec, $\left.99 \%\right)$, bromato de sódio $\left(\mathrm{NaBrO}_{3}\right.$, Vetec, $\left.99,5 \%\right)$, periodato de sódio $\left(\mathrm{NaIO}_{4}\right.$, Carlo Erba, $100 \%$, ), persulfato de potássio $\left(\mathrm{K}_{2} \mathrm{~S}_{2} \mathrm{O}_{8}\right.$, Synth, $98 \%$, P.A.), oxigênio $\left(\mathrm{O}_{2}\right.$, AGA $\left., 99,5 \%\right)$ e nitrogênio $\left(\mathrm{N}_{2}, \mathrm{AGA}, 99,999 \%\right)$ utilizados foram de grau analítico. As soluções foram preparadas usando água ultrapura obtida através do ultrapurificador Elga modelo USF CE. 


\section{Procedimento}

Os experimentos foram realizados em reator de vidro borossilicato $(200 \mathrm{~mL})$, com parede dupla para circulação de água proveniente do banho termostático com refrigeração, garantindo a permanência da temperatura em $30,0 \pm 0,1^{\circ} \mathrm{C}$ com reator aberto à atmosfera, a não ser que seja mencionado o contrário. Quantidades adequadas dos oxidantes $\mathrm{NaClO}_{3}, \mathrm{NaBrO}_{3}, \mathrm{NaIO}_{4}$ e $\mathrm{K}_{2} \mathrm{~S}_{2} \mathrm{O}_{8}$ foram adicionadas respectivamente a $150 \mathrm{~mL}$ tanto da solução de azo corante quanto da suspensão de $\mathrm{ZnO}$ contendo azo corante. A suspensão formada por acid orange 7 e óxido de zinco foi agitada em $600 \mathrm{rpm}$ (Fisatom 752 ) por 60 min no escuro, para permitir a adsorção do corante na superfície do fotocatalisador. A suspensão e a solução foram saturadas ou não com ar, oxigênio ou nitrogênio durante a irradiação com bolhas pequenas. O potencial do sistema foi medido usando-se o eletrodo de ORP (Digimed, DM10) à temperatura ambiente. A irradiação foi feita no interior de uma câmara de irradiação usando a lâmpada de vapor de $\mathrm{Hg}$ sem bulbo de $125 \mathrm{~W}$. A irradiância correspondente a 195,00 $\pm 20,00 \mu \mathrm{W} \mathrm{cm}{ }^{-2}$ foi medida por radiômetro (Topcon UVR-2) com o reator aberto ou fechado. Alíquotas de $1,1 \mathrm{~mL}$ foram coletadas e filtradas (Millipore-0,22 $\mu \mathrm{m}$ ) em tempos pré-determinados e medido o pH (Marte MB-10) durante a irradiação. A descoloração do AO7 $\left(\varepsilon=1,51 \times 10^{4} \mathrm{~L} \mathrm{~mol}^{-1} \mathrm{~cm}^{-1}\right)$ foi monitorada em $485 \mathrm{~nm}$ espectrofotometricamente (Hitachi U-3000) a $30^{\circ} \mathrm{C}$.

As constantes de velocidade de descoloração, $k_{o b s}$, foram determinadas a partir da inclinação $(r \geq 0,991, \mathrm{~s} \leq 0,109)$ de $\ln$ da concentração de $\mathrm{AO} 7$ em função do tempo de irradiação, respectivamente, para fotocatálise em reator aberto/fechado. Para fotólise em reator aberto/fechado o coeficiente de correlação foi menor ( $\mathrm{r} \geq 0,974, \mathrm{~s} \leq$ $0,065)$. As $k_{o b s}$ foram reprodutíveis em $\pm 5 \%$. As medidas foram feitas em duplicata e, quando necessárias, em triplicata.

\section{RESULTADOS E DISCUSSÃO}

\section{Descoloração de acid orange 7 irradiado}

A descoloração de acid orange $7\left(0,50 \times 10^{-4} \mathrm{~mol} \mathrm{~L}^{-1}\right)$ em suspensão de $\mathrm{ZnO}\left(1,5 \mathrm{~g} \mathrm{~L}^{-1}\right)$ em função do tempo de irradiação (Figura 2) seguiu o comportamento cinético de $1^{\mathrm{a}}$ ordem em $\mathrm{pH}$ natural de 6,9 a $30^{\circ} \mathrm{C}$. As bandas em 485 e $430 \mathrm{~nm}$ correspondem, respectivamente, às formas hidrazona e azo do corante, originadas da interação tautomérica da ligação de hidrogênio entre os oxigênios do grupo naftila e o hidrogênio-b da ligação-azo correspondente, ${ }^{23}$ que funcionam como sítios ativos para o ataque oxidativo. ${ }^{24} \mathrm{~A}$ diminuição das bandas com absorções máximas em 228, 255 e 307 nm e do intermediário em $347 \mathrm{~nm}$ formado em 20 min de irradiação, de modo similar ao processo $\mathrm{UV} / \mathrm{H}_{2} \mathrm{O}_{2},{ }^{25}$ indica o desaparecimento dos grupos aromáticos e, consequentemente, a degradação de $\mathrm{AO} 7$ em 90 min. O pH no final da irradiação tornou-se levemente mais alcalino aumentando de 7,0 para 7,4.

As constantes de velocidade de descoloração, obtidas por fotocatálise mediada por óxido de zinco na ausência dos oxidantes, foram equivalentes a $14,33 \times 10^{-3}(\mathrm{r}=0,992 ; \mathrm{s}=0,078)$ e $23,15 \times 10^{-3} \mathrm{~min}^{-1}$ $(\mathrm{r}=0,991 ; \mathrm{s}=0,108)$, respectivamente, em reator fechado e aberto à atmosfera a $30^{\circ} \mathrm{C}$, indicando que o ar sobre a superfície interfere na descoloração. A concentração de $1,5 \mathrm{~g} \mathrm{~L}^{-1}$ de $\mathrm{ZnO}$, utilizada neste trabalho, não impede a penetração de luz, pois se verificou que na descoloração de $1,0 \times 10^{-4} \mathrm{~mol} \mathrm{~L}^{-1}$ de $\mathrm{AO} 7$, as constantes de velocidade foram respectivamente iguais a $10,41 \times 10^{-3} \mathrm{~min}^{-1}(\mathrm{r}=0,997 ; \mathrm{s}=0,057)$ para $1,5 \mathrm{~g} \mathrm{~L}^{-1}$ e $20,16 \times 10^{-3} \mathrm{~min}^{-1}(\mathrm{r}=0,998 ; \mathrm{s}=0,042)$ para $2,5 \mathrm{~g} \mathrm{~L}^{-1} \mathrm{de}$ $\mathrm{ZnO}$. Além disso, é importante mencionar que a descoloração de acid orange 7 foi pouco significativa $\left(9,27 \times 10^{-5} \mathrm{~min}^{-1}, \mathrm{r}=0,883 ; \mathrm{s}=0,007\right)$ por fotólise direta realizada em reator aberto durante $7 \mathrm{~h}$ a $30^{\circ} \mathrm{C}$.

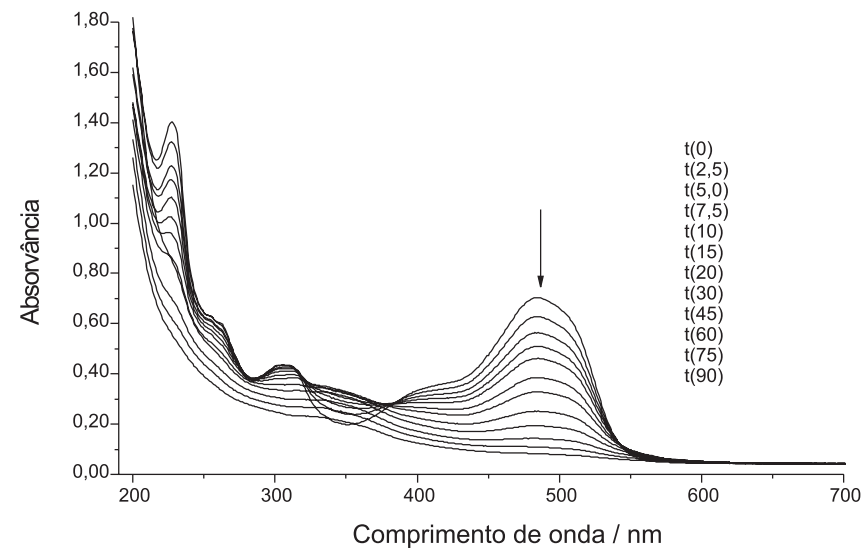

Figura 2. Variações espectrais na descoloração e degradação de acid orange $7\left(0,50 \times 10^{-4} \mathrm{~mol} \mathrm{~L}^{-1}\right)$ em suspensão aquosa de $\mathrm{ZnO}\left(1,5 \mathrm{~g} \mathrm{~L}^{-1}\right)$ em função do tempo de irradiação em $\mathrm{pH}$ natural a $30^{\circ} \mathrm{C}$

\section{Fotocatálise de acid orange 7 na presença de oxidantes}

A habilidade de um semicondutor, sob iluminação, aumentar a velocidade de degradação de um composto orgânico é atribuída à estrutura eletrônica, caracterizada pelas bandas de valência completa e de condução, vazia. Ao irradiar o óxido de zinco com energia igual ou maior que a energia de band gap $(3,2 \mathrm{eV})$, os elétrons da banda de valência são excitados à banda de condução para produzir os pares elétrons-buracos (Equação 1). ${ }^{6}$

$\mathrm{ZnO} \stackrel{h v}{\longrightarrow} \mathrm{e}^{-}+\mathrm{h}^{+}$

Estes pares podem ou recombinar e dissipar a energia como calor, ou migrar à superfície do semicondutor e participar das reações de oxidação-redução interfaciais através da formação do radical hidroxila, 'OH (Equação 2) e do ânion radical superóxido, $\mathrm{O}_{2}^{\cdot \bullet}$ (Equação 3).

$$
\begin{aligned}
& \mathrm{h}^{+}+\mathrm{H}_{2} \mathrm{O} \rightarrow \bullet \mathrm{OH}+\mathrm{H}^{+} \\
& \mathrm{e}^{-}+\mathrm{O}_{2} \rightarrow \mathrm{O}_{2}{ }^{\cdot-}
\end{aligned}
$$

$\mathrm{O}$ ânion radical superóxido na presença de $\mathrm{H}_{2} \mathrm{O}$ é convertido em radical hidroxila (Equação 4) que degrada o corante.

$\mathrm{O}_{2}^{\cdot-}+\mathrm{H}_{2} \mathrm{O} \rightarrow \cdot \cdot \mathrm{OH}+\mathrm{OH}^{-}+1 / 2 \mathrm{O}_{2}$

Por outro lado, a adição de oxidantes promove o aumento da eficiência quântica pela inibição da recombinação do par elétronburaco na superfície do semicondutor assim como o processo térmico e fotoquímico na suspensão, ${ }^{26-28}$ aumentando a constante de velocidade de degradação. Dentre os oxidantes adicionados, o persulfato apresentou o melhor desempenho em aumentar a constante de velocidade de descoloração. Esta propriedade foi menor para o periodato, seguida pelo bromato e, por último, para o clorato em reator aberto, quando se variou a concentração de $2,0 \times 10^{-4}$ a $2,0 \times 10^{-1} \mathrm{~mol} \mathrm{~L}^{-1} \mathrm{em} \mathrm{pH}$ natural a $30{ }^{\circ} \mathrm{C}$ conforme mostrado na Figura 3. A adição de $2,0 \times 10^{-4} \mathrm{~mol} \mathrm{~L}^{-1}$ de $\mathrm{ClO}_{3}^{-}$à suspensão de $\mathrm{ZnO}$ contendo o azo corante elevou a constante de velocidade para $26,51 \times 10^{-3} \mathrm{~min}^{-1}(\mathrm{r}=0,991 ; \mathrm{s}=0,096)$. Este aumento foi atribuído ao fato de o íon clorato (Equação 5), o radical $\mathrm{ClO}_{2}$ (Equação 6) e espécies como $\mathrm{HClO}_{2}$ e $\mathrm{HClO}$ (Equações 7 e 8$)^{29}$ aceitarem prontamente os elétrons da banda de condução para oxidar a molécula de acid orange 7. 
$\mathrm{ClO}_{3}^{-}+2 \mathrm{H}^{+}+\mathrm{e}_{\mathrm{bc}}^{-} \rightarrow \mathrm{ClO}_{2}^{-}+\mathrm{H}_{2} \mathrm{O}$

$\mathrm{ClO}_{2}^{\cdot}+\mathrm{H}^{+}+\mathrm{e}_{\mathrm{bc}}^{-} \rightarrow \mathrm{HClO}_{2}$

$\mathrm{HClO}_{2}+2 \mathrm{H}^{+}+2 \mathrm{e}_{\mathrm{bc}}^{-} \rightarrow \mathrm{HClO}+\mathrm{H}_{2} \mathrm{O}$

$\mathrm{HClO}+\mathrm{H}^{+}+2 \mathrm{e}_{\mathrm{bc}}^{-} \rightarrow \mathrm{Cl}^{-}+\mathrm{H}_{2} \mathrm{O}$

A constante de velocidade aumentou até $30,96 \times 10^{-3} \mathrm{~min}^{-1}(\mathrm{r}=$ 0,994; $\mathrm{s}=0,07)$, quando se adicionou $2,5 \times 10^{-3} \mathrm{~mol} \mathrm{~L}^{-1}$ de clorato. A diminuição para $27,76 \times 10^{-3}(\mathrm{r}=0,991 ; \mathrm{s}=0,108)$ e $22,14 \times 10^{-3} \mathrm{~min}^{-1}$ $(\mathrm{r}=0,991 ; \mathrm{s}=0,103)$ com a adição, respectivamente, de $12,50 \times 10^{-2}$ e $20,00 \times 10^{-2} \mathrm{~mol} \mathrm{~L}^{-1}$ de $\mathrm{NaClO}_{3}$ conforme pode ser observada na Figura 3 é atribuída à saturação do oxidante na superfície do semicondutor.

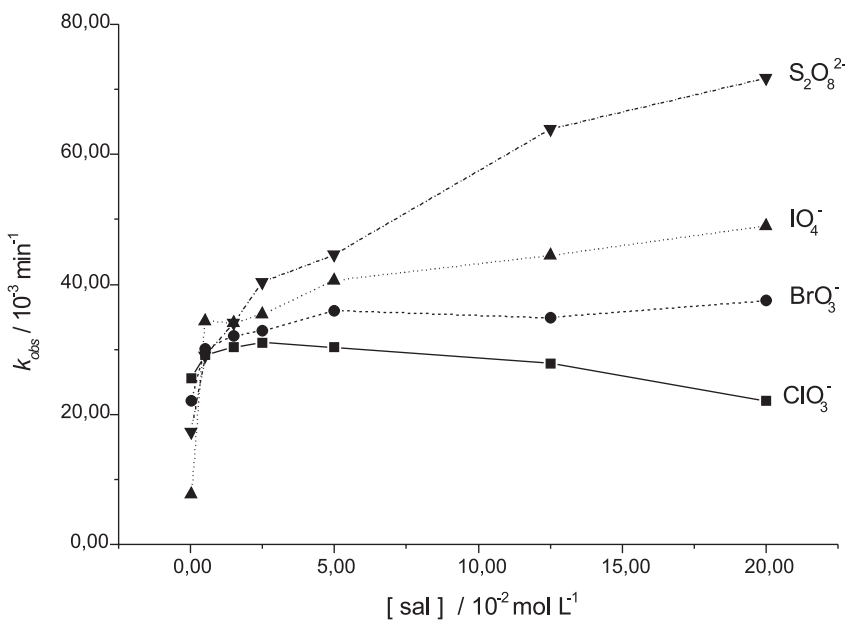

Figura 3. Constante de velocidade de descoloração, $k_{o b s}$, de AO7 $\left(0,50 \times 10^{-4}\right.$ mol $\left.\mathrm{L}^{-1}\right)$ em suspensão de $\mathrm{ZnO}\left(1,5 \mathrm{~g} \mathrm{~L}^{-1}\right)$ na presença de oxidantes como (-) $\mathrm{ClO}_{3}^{-}(\bullet) \mathrm{BrO}_{3}^{-}(\mathbf{\Delta}) \mathrm{IO}_{4}^{-}$e ( $\left.\mathbf{\nabla}\right) \mathrm{S}_{2} \mathrm{O}_{8}^{2-}$ sob radiação $\mathrm{UV}$ em $\mathrm{pH}$ natural a $30^{\circ} \mathrm{C}$

Por outro lado, ao adicionar $2,0 \times 10^{-4} \mathrm{~mol} \mathrm{~L}^{-1}$ de bromato, a constante de velocidade inicial foi da mesma ordem de grandeza do clorato, ou seja, $22,07 \times 10^{-3} \mathrm{~min}^{-1}(\mathrm{r}=0,991 ; \mathrm{s}=0,109)$ e aumentou para $35,88 \times 10^{-3} \mathrm{~min}^{-1}(\mathrm{r}=0,998 ; \mathrm{s}=0,053)$ em $5,00 \times 10^{-2} \mathrm{~mol} \mathrm{~L}^{-1}$ mantendo-se praticamente constante até $20,00 \times 10^{-2} \mathrm{~mol} \mathrm{~L}^{-1}$ de bromato conforme pode ser observada na Figura 3. De forma similar ao íon clorato, isto pode ser atribuído à reação entre o íon $\mathrm{BrO}_{3}^{-}$e o elétron da banda de condução, formando o radical $\mathrm{BrO}_{2} \cdot$ e outras espécies reativas até produzir o íon $\mathrm{Br}$, que reage com água para formar o íon hidroxila e, na sequência, o radical hidroxila, responsável pela descoloração do azo corante. Considerando-se que os potenciais de redução de clorato e bromato sejam equivalentes a $1,15 \mathrm{~V},{ }^{29}$ as maiores constantes de velocidade de descoloração em meio de bromato podem ser atribuídas predominantemente à menor energia de ligação entre $\mathrm{Br}-\mathrm{O}\left(235,1 \pm 0,4 \mathrm{~kJ} \mathrm{~mol}^{-1}\right)$ quando comparada a $\mathrm{Cl}-\mathrm{O}$ $\left(269,1 \mathrm{~kJ} \mathrm{~mol}^{-1}\right) .^{30}$

A adição do íon periodato de $2,0 \times 10^{-4}$ a $5,0 \times 10^{-2} \mathrm{~mol} \mathrm{~L}^{-1}$ aumentou mais ainda a constante de velocidade, ou seja, de $21,76 \times 10^{-3}(\mathrm{r}=$ $0,991 ; \mathrm{s}=0,105)$ a $48,94 \times 10^{-3} \mathrm{~min}^{-1}(\mathrm{r}=0,998 ; \mathrm{s}=0,068)$, atribuída à menor energia de dissociação da ligação entre o iodo e o oxigênio $\left(180 \mathrm{~kJ} \mathrm{~mol}^{-1}\right)^{30}$ e maior eficiência na captura do elétron para formar espécies reativas como $\mathrm{IO}_{3} \cdot \mathrm{HIO}_{3}, \mathrm{HIO}_{2}, \mathrm{HIO}$ e, subseqüentemente, o radical hidroxila. $\mathrm{O}$ fato de, em concentrações mais baixas, as constantes de velocidade possuirem a mesma ordem de grandeza, sugere que as moléculas de corante estariam adsorvidas em sítios relativamente distantes na superfície do semicondutor. Isto implica que a colisão efetiva seja menos provável entre a molécula de azo corante e o fóton com energia de band gap, isto é, quanto menor a concentração do oxidante independentemente de sua natureza, menor será a constante de velocidade, justificando assim o resultado obtido. À medida que os sítios forem ocupados com o aumento da concentração de oxidante, a frequência de colisão será maior e, consequentemente, a formação de espécies que capturam os elétrons da banda de condução e aumentam a constante de velocidade. ${ }^{31}$ Sadik $^{32}$ ao irradiar $0,50 \times 10^{-4} \mathrm{~mol} \mathrm{~L}^{-1} \mathrm{de}$ AO7 em suspensão de $0,5 \mathrm{~g} \mathrm{~L}^{-1} \mathrm{de}^{\mathrm{TiO}_{2}}$, a constante de velocidade de descoloração aumentou de $6,1 \times 10^{-2}$ a $74,74 \times 10^{-2} \mathrm{~min}^{-1}$ com a adição de $4,67 \times 10^{-5}$ a $1,0 \times 10^{-3} \mathrm{~mol} \mathrm{~L}^{-1}$ de $\mathrm{IO}_{4}^{-}$, indicando que a captura os elétrons ejetados pelo dióxido de titânio ocorre bem mais rapidamente do que em óxido de zinco.

Por outro lado, a adição de persulfato no meio reacional quadruplicou a constante de velocidade de descoloração de $17,25 \times 10^{-3}$ $(\mathrm{r}=0,992 ; \mathrm{sD}=0,104)$ a $71,75 \times 10^{-3} \mathrm{~min}^{-1}(\mathrm{r}=0,993 ; \mathrm{s}=0,084)$ no intervalo de $2,0 \times 10^{-4}$ a $20,0 \times 10^{-2} \mathrm{~mol} \mathrm{~L}^{-1} \mathrm{em} \mathrm{pH}$ natural a $30^{\circ} \mathrm{C}$. Isto pode ser justificado, porque o elétron fotogerado pode ser capturado pelo íon persulfato para formar o ânion radical sulfato $\mathrm{SO}_{4}{ }^{-}$(Equação 9), um agente oxidante forte com potencial redox de $2,6 \mathrm{~V},{ }^{33}$ que pode abstrair um átomo de hidrogênio de $\mathrm{H}_{2} \mathrm{O}$ ou de uma molécula orgânica para produzir o radical hidroxila (Equação 10) e o oxigênio molecular (Equação 11). Além disso, a energia de dissociação da ligação simples oxigênio-oxigênio $\left(146 \mathrm{~kJ} \mathrm{~mol}^{-1}\right)^{34}$ é menor que a de halogênio-oxigênio dos outros oxidantes investigados. ${ }^{30}$

$\mathrm{S}_{2} \mathrm{O}_{8}{ }^{2-}+\mathrm{e}_{\mathrm{bc}}^{-} \rightarrow \mathrm{SO}_{4}^{--}+\mathrm{SO}_{4}^{2-}$

$\mathrm{SO}_{4}^{--}+\mathrm{H}_{2} \mathrm{O} \rightarrow \cdot \mathrm{OH}+\mathrm{SO}_{4}^{2-}+\mathrm{H}^{+}$

$4 \mathrm{SO}_{4}^{-{ }^{-}}+2 \mathrm{H}_{2} \mathrm{O} \rightarrow 4 \mathrm{SO}_{4}{ }^{2-}+4 \mathrm{H}^{+}+\mathrm{O}_{2}$

Conforme mostrado na Figura 3, dos quatro oxidantes utilizados para descolorir o AO7, o persulfato foi, sem dúvida, aquele que apresentou melhor desempenho nas mesmas condições experimentais, especialmente devido à formação do radical ânion sulfato e por consequência, o radical hidroxila (Equação 10) e o oxigênio molecular.

A partir destes resultados conclui-se que a constante de velocidade de descoloração de AO7 depende basicamente da capacidade de captura, pelo agente oxidante, do elétron disponível na superfície do catalisador e produzido pela incidência de radiação, a fim de evitar a recombinação do par elétron-lacuna. Isto justifica o rápido aumento da constante de velocidade em concentrações mais baixas e um aumento relativamente mais lento a partir de $0,5 \times 10^{-4} \mathrm{~mol} \mathrm{~L}^{-1}$ de oxidante, na qual se supõe que a proporção de sítios disponíveis seja pequena. Além disso, a menor eficiência pode ser consequência de um processo de fotólise do corante, prejudicado pelo $\mathrm{ZnO}$, independentemente da presença do oxidante.

\section{Fotólise de acid orange 7 na presença de oxidantes}

A variação das constantes de velocidade de descoloração de $\mathrm{AO} 7$

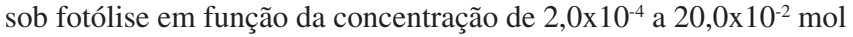
$\mathrm{L}^{-1}$ dos quatro oxidantes inorgânicos a $30^{\circ} \mathrm{C}$ é mostrada na Figura 4. A adição de íon clorato inibiu a descoloração neste intervalo, pois a reação foi bem mais lenta em toda extensão $\left(1,10 \times 10^{-4} \mathrm{~min}^{-1}, \mathrm{r}=\right.$ 0,$974 ; \mathrm{s}_{\mathrm{D}}=0,003$ a $\left.2,79 \times 10^{-3} \mathrm{~min}^{-1}, \mathrm{r}=0,986 ; \mathrm{s}=0,019\right)$ em relação à fotocatálise. Por outro lado, o corante descoloriu mais rapidamente $\left(8,46 \times 10^{-4} \mathrm{~min}^{-1}, \mathrm{r}=0,975 ; \mathrm{s}=0,018\right)$ com a adição de $2,0 \times 10^{-4}$ mol L-1 de bromato e aumentou gradualmente até $40,68 \times 10^{-3} \mathrm{~min}^{-1}$ $(\mathrm{r}=0,994 ; \mathrm{s}=0,063)$, tornando-se mais rápida que a fotocatálise 
$\left(37,49 \times 10^{-3} \mathrm{~min}^{-1}(\mathrm{r}=0,992 ; \mathrm{s}=0,062)\right)$ ao adicionar $20,0 \times 10^{-2} \mathrm{~mol}$ $\mathrm{L}^{-1}$ de bromato. As constantes de velocidade de descoloração de AO7 aumentaram de forma mais efetiva, quando se adicionou o periodato e o persulfato, pois a descoloração se tornou mais rápida, por fotólise, a partir de $1,5 \times 10^{-2} \mathrm{~mol} \mathrm{~L}^{-1}$. Na presença do primeiro, as constantes de velocidade variaram de $6,06 \times 10^{-3}(\mathrm{r}=0,988 ; \mathrm{s}=0,025)$ a $8,33 \times 10^{-2}$ $\min ^{-1}(r=0,997 ; s=0,064)$ enquanto que na de persulfato de $7,2 \times 10^{-4}$ $(\mathrm{r}=0,989 ; \mathrm{s}=0,007)$ a $1,32 \times 10^{-1} \min ^{-1}(\mathrm{r}=0,983 ; \mathrm{s}=0,010)$ como mostrado na Figura 4.

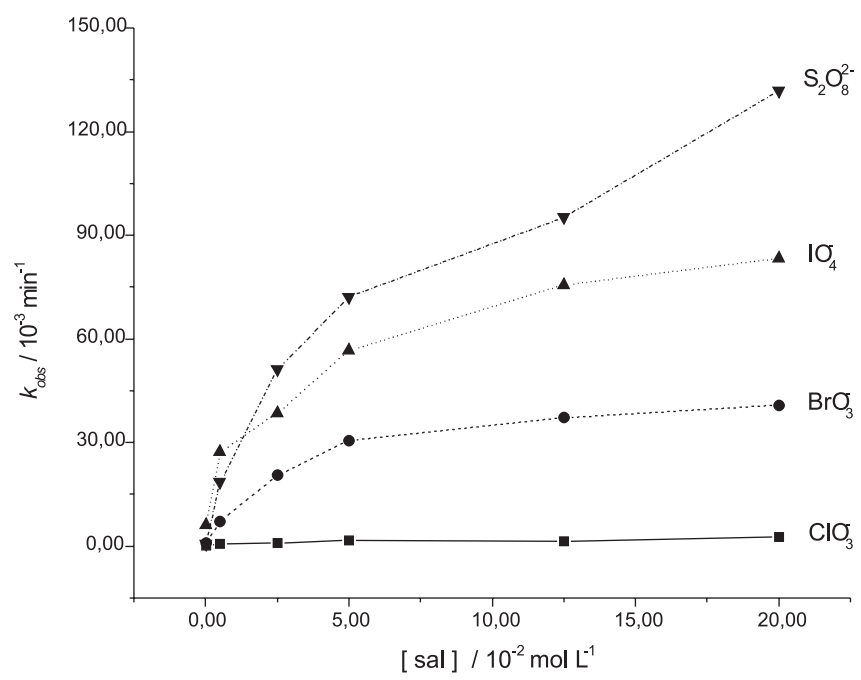

Figura 4. Constante de velocidade de descoloração, $\mathrm{k}_{\mathrm{obs}}$, de acid orange 7 $\left(0,50 \times 10^{-4} \mathrm{~mol} \mathrm{~L}^{-1}\right)$ sob fotólise em função da concentração de oxidantes como (•) $\mathrm{ClO}_{3}^{-}(\bullet) \mathrm{BrO}_{3}^{-}(\mathbf{\Delta}) \mathrm{IO}_{4}^{-} e(\mathbf{\nabla}) \mathrm{S}_{2} \mathrm{O}_{8}^{2-}$ em pH natural a $30^{\circ} \mathrm{C}$

A adição de clorato foi menos efetiva na descoloração de AO7 em relação aos outros oxidantes na fotólise, apesar de ter aumentado a constante de velocidade por aproximadamente 25 vezes, ou seja, de $0,11 \times 10^{-3}$ a $2,79 \times 10^{-3} \mathrm{~min}^{-1}$, quando se variou a concentração de

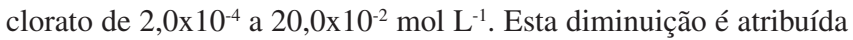
à menor quantidade dos radicais $\mathrm{ClO}_{2}{ }^{\circ} \mathrm{e} \mathrm{O}^{-*}$ produzida por fotólise (Equação 12) devido à maior energia de ligação entre os átomos de cloro e oxigênio, quando comparada às de bromo-oxigênio e iodooxigênio. ${ }^{30}$ Assim, pode ser inferido que o número de radical hidroxila formado em meio ácido é relativamente pequeno (Equação 13).

$\mathrm{ClO}_{3} \stackrel{h \mathrm{v}}{\longrightarrow} \mathrm{ClO}_{2}{ }^{-}+\mathrm{O}^{\cdot}$

$\mathrm{O}^{*}+\mathrm{H}^{+} \rightarrow \bullet \cdot \mathrm{OH}$

A diferença entre as constantes de velocidade de descoloração de AO7 utilizando-se o íon clorato $2,0 \times 10^{-4} \mathrm{~mol} \mathrm{~L}^{-1}$ na fotocatálise $\left(26,51 \times 10^{-3} \mathrm{~min}^{-1}\right)$ e na fotólise $\left(0,11 \times 10^{-3} \mathrm{~min}^{-1}\right)$ é, principalmente, devido aos sítios disponíveis para adsorção do azo corante e da água na superfície do semicondutor durante a irradiação para produção de radical hidroxila. $\mathrm{O}$ radical $\mathrm{ClO}_{2}$ é reduzido em solução aquosa sucessivamente a $\mathrm{HClO}_{2}, \mathrm{HClO} \mathrm{Cl}^{-}$de modo similar ao mostrado nas Equações 5-8. A reação total é então dada por:

$\mathrm{ClO}_{3}^{-}+6 \mathrm{e}_{\text {aq }}^{-}+6 \mathrm{H}^{+} \rightarrow \mathrm{Cl}^{-}+3 \mathrm{H}_{2} \mathrm{O}$

A adição de $2,0 \times 10^{-4} \mathrm{~mol} \mathrm{~L}^{-1}$ de bromato de sódio aumentou a constante de velocidade de descoloração de $\mathrm{AO} 7$ para $0,85 \times 10^{-3}$

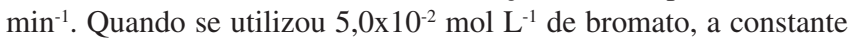
de velocidade aumentou para $30,63 \times 10^{-3} \mathrm{~min}^{-1}(\mathrm{r}=0,998 ; \mathrm{s}=0,056)$ e em $20,0 \times 10^{-2} \mathrm{~mol} \mathrm{~L}^{-1}$ a descoloração foi mais rápida $\left(40,68 \times 10^{-3}\right.$ $\left.\min ^{-1}\right)$ do que a fotocatálise $\left(37,49 \times 10^{-3} \min ^{-1}(r=0,992 ; \mathrm{s}=0,061)\right)$. Isto mostra que o aumento na concentração de bromato favorece a produção de radicais como $\mathrm{BrO}_{2} \cdot \mathrm{e} \mathrm{O}^{*} \mathrm{e}$, consequentemente, a produção de radical hidroxila e a descoloração do azo corante.

Na presença de $\mathrm{IO}_{4}^{-} 2,0 \times 10^{-4} \mathrm{~mol} \mathrm{~L}^{-1}$ a constante de velocidade de descoloração do azo corante foi ainda maior, ou seja, de $6,06 \times 10^{-3}$ $\min ^{-1}$, ao redor de 55 e 7 vezes respectivamente maiores em relação ao clorato e bromato. A constante de velocidade de descoloração por fotólise tornou-se maior $\left(36,37 \times 10^{-3} \mathrm{~min}^{-1}(\mathrm{r}=0,999 ; \mathrm{s}=0,032)\right)$ que a fotocatálise $\left(34,10 \times 10^{-3} \mathrm{~min}^{-1}(\mathrm{r}=0,975 ; \mathrm{s}=0,060)\right)$ para $1,5 \times 10^{-2} \mathrm{~mol}$ $\mathrm{L}^{-1}$ de periodato. Este comportamento foi atribuído à maior reatividade dos radicais produzidos a partir do periodato como $\mathrm{IO}_{3} \cdot \mathrm{HIO}_{3}, \mathrm{HIO}_{2}$ e HIO, assim como à menor energia de ligação de iodo-oxigênio em relação aos dois outros. ${ }^{30}$

A adição de $2,0 \times 10^{-4} \mathrm{~mol} \mathrm{~L}^{-1}$ de persulfato resultou em uma constante de velocidade de descoloração de $0,72 \times 10^{-3} \mathrm{~min}^{-1}$ com reator aberto a $30^{\circ} \mathrm{C}$. Apesar de este valor ser menor comparado ao periodato, a capacidade de oxidação aumentou com o aumento da concentração sob irradiação, pois se adicionando $1,0 \times 10^{-2} \mathrm{~mol} \mathrm{~L}^{-1}$ de persulfato a constante de velocidade atingiu $34,35 \times 10^{-3} \mathrm{~min}^{-1}(\mathrm{r}=0,980 ; \mathrm{s}=0,056)$, valor este maior que a de fotocatálise $\left(31,95 \times 10^{-3} \mathrm{~min}^{-1}, \mathrm{r}=0,995\right.$; $\mathrm{s}=0,063)$ que aumentou gradativamente até $131,75 \times 10^{-3} \mathrm{~min}^{-1}$ para $20,0 \times 10^{-2} \mathrm{~mol} \mathrm{~L}^{-1}$ do oxidante. A maior facilidade na remoção de cor por fotólise em relação à fotocatálise a partir de uma dada concentração de oxidante sugere que, quanto maior a concentração de oxidante, maior é a produção das espécies reativas mencionadas, ao contrário da degradação fotocatalítica em que, entre outros fatores, diversos estudos têm mostrado que o número de sítios disponíveis, sob iluminação, na superfície do semicondutor é crucial na conversão. ${ }^{14-20,24,28}$ A exceção ocorreu para o íon clorato para o qual a energia de ligação entre os átomos de oxigênio e cloro é relativamente grande. Consequentemente, o aumento na constante de velocidade de descoloração de AO7 por fotólise em meio de persulfato é devido ao rendimento quântico equivalente a 2 , resultante da ruptura da ligação simples $\mathrm{O}-\mathrm{O}$ e da formação do ânion radical sulfato $\mathrm{SO}_{4}{ }^{-}$- de acordo com a Equação 15, ${ }^{35}$ assim como por redução em meio aquoso (Equação 16) que, por ser instável, sofre hidrólise, dando origem ao radical hidroxila de acordo com a Equação 10.

$\mathrm{S}_{2} \mathrm{O}_{8}{ }^{2-}+\mathrm{hv} \rightarrow 2 \mathrm{SO}_{4}{ }^{--}$
$\mathrm{S}_{2} \mathrm{O}_{8}{ }^{2-}+\mathrm{e}^{-}{ }_{\mathrm{aq}}^{-} \rightarrow \mathrm{SO}_{4}{ }^{--}+\mathrm{SO}_{4}{ }^{2-}$

\section{Efeito do oxigênio dissolvido na descoloração de AO7}

O efeito do oxigênio dissolvido foi investigado, comparando-se as constantes de velocidade de descoloração de $\mathrm{AO} 7$ na presença de persulfato $2,5 \times 10^{-2} \mathrm{~mol} \mathrm{~L}^{-1}$ a $30{ }^{\circ} \mathrm{C}$, sob fotocatálise e fotólise, saturando-se ou não com ar, com $\mathrm{O}_{2}$ e $\mathrm{N}_{2}$ respectivamente em reator fechado e aberto à atmosfera. É importante mencionar que não houve variação de irradiância, dentro dos erros experimentais, da lâmpada de vapor de $\mathrm{Hg}$ com reator aberto ou fechado. De acordo com a Tabela 1 as maiores constantes de velocidade de reação ocorreram em reator aberto, atribuídas à maior disponibilidade de ar sobre a superfície da solução ou suspensão de $\mathrm{ZnO}$ para promover a oxidação.

Tang e $\mathrm{Chen}^{36}$ estudaram o efeito do oxigênio dissolvido na degradação do azocorante reactive black 5 através do processo $\mathrm{TiO}_{2} / \mathrm{UV}$ em três condições, ou seja, com o reator aberto à atmosfera, com saturação de ar e de $\mathrm{O}_{2}$, respectivamente. A descoloração foi mais rápida com o sistema saturado de $\mathrm{O}_{2}$, seguida pelas duas outras que apresentaram praticamente o mesmo resultado. Por outro lado, Habibi et al. ${ }^{37} \mathrm{ob}-$ 
Tabela 1. Constante de velocidade de descoloração, $k_{o b s}$, de AO7 em meio de persulfato sob fotocatálise mediada por óxido de zinco e fotólise em reatores aberto e fechado a $30^{\circ} \mathrm{C}$

\begin{tabular}{|c|c|c|c|c|c|c|c|}
\hline \multirow[b]{2}{*}{ Processo } & \multirow[b]{2}{*}{ Reator } & \multirow[b]{2}{*}[\mathrm{ZnO}]{$/ \mathrm{g} \mathrm{L}^{-1}$} & \multirow[b]{2}{*}{$\begin{array}{c}{\left[\mathrm{S}_{2} \mathrm{O}_{8}{ }^{2-}\right] /} \\
10^{-2} \mathrm{~mol} \mathrm{~L}^{-1}\end{array}$} & \multicolumn{4}{|c|}{$k_{o b s} / 10^{-3} \mathrm{~min}^{-1}$} \\
\hline & & & & $\begin{array}{c}\text { sem } \\
\text { satur. ar }\end{array}$ & $\begin{array}{c}\text { com } \\
\text { satur. ar }\end{array}$ & $\begin{array}{c}\text { com } \\
\text { satur. } \mathrm{O}_{2}\end{array}$ & $\begin{array}{c}\text { com } \\
\text { satur. } \mathrm{N}_{2}\end{array}$ \\
\hline Fotocatálise & aberto & 1,5 & 2,5 & 40,27 & 36,43 & 44,59 & 28,33 \\
\hline Fotocatálise & fechado & 1,5 & 2,5 & 29,29 & 13,39 & --- & --- \\
\hline Fotólise & aberto & --- & 2,5 & 51,23 & 40,07 & 56,66 & 30,78 \\
\hline Fotólise & fechado & --- & 2,5 & 6,86 & 4,22 & --- & --- \\
\hline
\end{tabular}

servaram que o efeito do oxigênio dissolvido não foi significativo na degradação fotocatalítica do diazocorante solofenil red 3BL (CI direct 80) na presença de $\mathrm{TiO}_{2}$. De acordo com a Tabela 1, as constantes de velocidade do sistema saturado de ar foram sistematicamente menores, tanto em reator fechado quanto em aberto, à atmosfera nos dois processos. Este comportamento aparentemente não é usual, já que o oxigênio presente no ar, em princípio, deveria contribuir no aumento da constante de velocidade na fotocatálise por evitar a recombinação do par elétron-lacuna, já que haveria a recepção do elétron pelo oxigênio. A mesma tendência seguida na fotólise sugere que estas moléculas não contribuiriam no aumento da constante de velocidade de descoloração. Para justificar estes resultados, a suspensão e a solução de acid orange 7 foram borbulhadas, durante a irradiação, respectivamente com oxigênio e desoxigenadas com nitrogênio em reator aberto. Além disso, a pequena diferença entre os potenciais de oxidação de $\mathrm{AO} 7$ na presença de persulfato, medidos em triplicata, respectivamente iguais a $498 \mathrm{mV}$ com borbulhamento de ar e de $491 \mathrm{mV}$ sem borbulhamento durante a fotólise, indica que a saturação de ar não aumenta a capacidade de oxidação do azo corante em meio de persulfato. Conforme a Tabela 1, as maiores constantes de velocidade de descoloração de AO7 em reator aberto sob fotólise e fotocatálise ocorreram quando foram saturadas com oxigênio e resultaram, respectivamente, em 56,66x $10^{-3}(\mathrm{r}=0,988$; $\mathrm{s}=0,140)$ e $44,59 \times 10^{-3} \mathrm{~min}^{-1}(\mathrm{r}=0,995 ; \mathrm{s}=0,067)$. Ao saturar com nitrogênio também em reator aberto, as constantes de velocidade foram menores e iguais a $30,78 \times 10^{-3} \mathrm{~min}^{-1}(\mathrm{r}=0,993 ; \mathrm{s}=0,102)$ para fotólise e $28,33 \times 10^{-3} \mathrm{~min}^{-1}(\mathrm{r}=0,998 ; \mathrm{s}=0,042)$ para fotocatálise. Consequentemente, a diminuição da constante de velocidade em meio saturado de ar de $40,27 \times 10^{-3}(\mathrm{r}=0,995 ; \mathrm{s}=0,066)$ para $36,43 \times 10^{-3} \mathrm{~min}^{-1}(\mathrm{r}=0,991$; $\mathrm{s}=0,103)$ por fotocatálise e de $51,23 \times 10^{-3}(\mathrm{r}=0,992 ; \mathrm{s}=0,101)$ para $40,07 \times 10^{-3} \mathrm{~min}^{-1}(\mathrm{r}=0,994 ; \mathrm{s}=0,090)$ por fotólise na presença de persulfato em reator aberto é um processo não totalmente esclarecido neste trabalho, mas no qual, aparentemente, as moléculas de oxigênio difundem à superfície da solução ou suspensão, impedindo a formação de espécies reativas. Estes resultados indicam que o monoazo corante AO7 pode ser descolorido em meio de persulfato mesmo na ausência de oxigênio dissolvido.

\section{CONCLUSÃO}

Este estudo mostrou que a constante de velocidade de descoloração de acid orange $7 \mathrm{em}$ pH natural $(6,9)$ a $30{ }^{\circ} \mathrm{C}$ aumenta com a adição de oxidantes como clorato, bromato, periodato e persulfato, tanto por fotocatálise mediada por $\mathrm{ZnO}$ quanto por fotólise, com exceção do clorato em reator aberto. A descoloração foi relativamente mais rápida em concentrações mais baixas de oxidantes e mais lenta em concentrações mais elevadas com exceção do persulfato na fotocatálise. Por outro lado, na fotólise a constante de velocidade de descoloração aumentou gradativamente, com exceção do clorato, ultrapassando valores obtidos por fotocatálise em concentrações a partir de $0,5 \times 10^{-4} \mathrm{~mol} \mathrm{~L}^{-1}$. Assim, o aumento relativamente pequeno da constante de velocidade no processo fotocatalítico foi atribuído à pequena proporção de sítios disponíveis e a diminuição, atribuída à saturação do oxidante na superfície do semicondutor. Além disso, a menor eficiência pode ser consequência de um processo de fotólise do corante, prejudicado pelo $\mathrm{ZnO}$, independentemente da presença do oxidante. A saturação de ar diminuiu as constantes de velocidade de descoloração de AO7, em meio de persulfato com saturação de ar, sob fotocatálise e fotólise, tanto no reator fechado como no aberto. Além disso, a descoloração de acid orange 7 independe de o oxigênio estar ou não dissolvido em meio de persulfato.

\section{AGRADECIMENTOS}

À Fundação Araucária pelo apoio financeiro na realização deste trabalho e ao Prof. Dr. W. J. Barreto pela valiosa discussão. A. C. Lucilha agradece a bolsa de Iniciação Científica PIBIC/CNPq.

\section{REFERÊNCIAS}

1. Zollinger, H.; Color Chemistry: Synthesis, Properties and Applications of Organic Dyes and Pigments, $2^{\text {nd }}$ ed. rev., VCH: Weinheim, 1991.

2. Guaratini, C. C. I.; Zanoni, M. V. B.; Quim. Nova 2000, 23, 71.

3. Konstantinou, I. K.; Albanis, T. A.; Appl. Catal., B 2004, 49, 1

4. Gogate, P. R.; Pandit, A. B.; Adv. Environ. Res. 2004, 8, 501.

5. Augugliaro, V.; Litter, M., Palmisano, L.; Soria, J.; J. Photochem. Photobiol., C 2006, 7, 127.

6. Hoffmann, M. R.; Martin, S. T.; Choi, W.; Bahnemann, D. W.; Chem. Rev. (Washington, DC, U.S.) 1995, 95, 69.

7. Legrini, O.; Oliveros, E.; Braun, A. M.; Chem. Rev. (Washington, DC, U.S.) $1993,93,671$

8. Malato, S.; Blanco, J.; Vidal, A.; Richter, C.; Appl. Catal., B 2002, 37, 1.

9. Pera-Titus, M.; Garcia-Molina, V.; Baños, M. A.; Gimenez, J.; Esplugas, S.; Appl. Catal., B 2004, 47, 219.

10. Jiu, J.; Isoda, S.; Adachi, M.; Wang, F.; J. Photochem. Photobiol., A 2007, 189, 314.

11. Uzunova, M.; Kostadinov, M.; Georgieva, J.; Dushkin, C.; Todorovsky, D.; Appl. Catal., B 2007, 73, 23.

12. Song, S.; Xu, L.; He, Z.; Chen, J.; Environ. Sci. Technol. 2007, 41, 5846.

13. Lizama, C.; Freer, J.; Baeza, J.; Mansilla, H. D.; Catal. Today 2002, 76, 235.

14. Sakthivel, S.; Neppolian, B.; Shankar, M. V.; Arabindoo, B.; Palanichamy, M.; Murugesan, V.; Sol. Energy Mater. Sol. Cells 2003, 77, 65.

15. Aykol, A.; Bayramoglu, M.; J. Hazard. Mater. 2005, 124, 241.

16. Pekakis, P. A.; Xekoukoulotakis, N. P.; Mantzavinos, D.; Water Res. 2006, 40, 1276.

17. Sobana, N.; Swaminathan, M.; Sep. Purif. Technol. 2007, 56, 101.

18. Jiang, Y.; Sun, Y.; Liu, H.; Zhu, F.; Yin, H.; Dyes Pigm. 2008, 78, 77.

19. Garcia, J. C.; Takashima, K.; J. Photochem. Photobiol., A 2003, 155, 215.

20. Ishiki, R. R.; Ishiki, H. M.; Takashima, K.; Chemosphere 2005, 58, 1461. 
21. Yassumoto, L.; Osajima, J. A.; Takashima, K.; Ecl. Quím. 2007, 32, 27. 22. Osajima, J. A.; Ishiki, H. M.; Takashima, K.; Monatsh. Chem. 2008, 139, 7.

23. Stylidi, M.; Kondarides, D. I., Verykios, X. E.; Appl. Catal., B 2004, 47, 189.

24. Daneshvar, N.; Salari, D.; Khataee, A. R.; J. Photochem. Photobiol., A 2003, 157, 111.

25. Behnajady, M. A.; Modirshahla, N.; Shokri, M.; Chemosphere 2004, 55, 129

26. Martin, S. T.; Lee, A. T.; Hoffmann, M. R.; Environ. Sci. Technol. 1995, 29, 2567.

27. Wang, Y.; Hong, C. S.; Water Res. 1999, 33, 2031.

28. Qamar, M.; Saquib, M.; Muneer, M.; Desalination 2005, 186, 255.
29. Epstein, I. R.; Kustin, K.; J. Phys. Chem. 1985, 89, 2275.

30. Lide, D. R.; Handbook of Chemistry and Physics, ed.-in-chief; $76^{\text {th }}$ ed, CRC: Boca Raton, 1995, chap. 9.

31. Clausen, D. N.; Takashima, K.; Quim. Nova 2007, 30, 1896

32. Sadik, W. A.; J. Photochem. Photobiol., A 2007, 191, 132.

33. Eberson, L.; Electron transfer reactions in Organic Chemistry, SpringerVerlag: Berlin, 1987.

34. Atkins, P.; de Paula, J.; Physical Chemistry, $8^{\text {th }}$ ed, Oxford: Oxford, 2006.

35. Dogliotti, L.; Hayon, E.; J. Phys. Chem. 1967, 71, 2511.

36. Tang, C.; Chen, V.; Water Res. 2004, 38, 2775.

37. Habibi, M. H.; Hassanzadeh, A.; Mahdavi, S.; J. Photochem. Photobiol., A 2005, 172, 89 . 